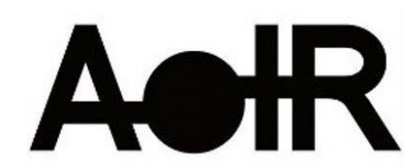

Selected Papers of \#AoIR2019:

The $20^{\text {th }}$ Annual Conference of the

Association of Internet Researchers

Brisbane, Australia / 2-5 October 2019

\title{
"WHAT WE CANNOT SPEAK ABOUT WE MUST TWEET": TRUST AMONG ISRAELI TWITTER USERS WITH DEPRESSION
}

\author{
Sofia Haytin \\ University of Haifa \\ Oren Livio \\ University of Haifa

\section{Theoretical Background}

Research on trust in online environments has demonstrated the centrality of trust for virtual sociality, the ways in which trust arises, and the consequences of both the emergence of trust and its associated risks (de Laat, 2008). While the affordances of different internet platforms lead to different manifestations of trust and personal exposure, it appears that despite customarily having little information about their potential audiences, participants in online environments such as blogs often assume others to be worthy of intimacy that is indeed reciprocated, resulting in a generalized climate of mutual trust (Smith, 2016). At the same time, the complexities of sharing private information lead individuals to different negotiations of the public/private divide, ranging from the avoidance of disclosing information and attempts to limit exposure to specific audiences, to more radical attempts to reveal everything in an act of "empowering exhibitionism" (Koskela, 2004).

A specific type of online environment that has been studied extensively is that of internet-based support groups for individuals suffering from various illnesses. Research on such groups has demonstrated that given high levels of sincerity and self-disclosure, they offer participants information, medical help, and emotional support (van UdenKraan et al., 2008). To date, most studies have focused on internet forums and social media groups devoted to specific physical diseases, and in which some forms of hierarchy exist (e.g., group moderators). In recent years, however, some studies have begun investigating the emergence of "communities"-of-sorts developing "naturally" in

Suggested Citation (APA): Haytin, S., \& Livio, O. (2019, October 2-5). "What we cannot speak about we must tweet": Trust among Israeli Twitter users with depression. Paper presented at AolR 2019: The 20th Annual Conference of the Association of Internet Researchers. Brisbane, Australia: AolR. Retrieved from http://spir.aoir.org. 
the more dispersed, unstructured, a-hierarchical spaces of social media platforms such as Twitter (Naslund et al., 2016).

In this study we examine the development of trust among Israeli Twitter users with depression. We seek to complicate existing research by studying an unstructured, spontaneously emerging "community" composed of individuals with a mental disorder, which has developed on a social media platform characterized by affordances different from those commonly examined to date. We also focus on a different national/cultural context than the frequently studied North American/European environments.

\section{Method}

Unlike the immensely popular Facebook ( $75 \%$ of Israelis are active users), Twitter in Israel (4.3\% penetration rate) has been largely ignored by social media scholars. It is precisely this perceived "failure," however, that has enabled it to become a breeding ground for self-defined outsiders, who have created a distinct discursive space in which non-hegemonic voices are more prominent.

Our study is based on in-depth, semi-structured interviews with eight Twitter users with depression (four women, four men, ages 21-47). Interviews were conducted face-toface, with the participants' anonymity guaranteed. Participants were asked about their social media practices and perceptions of Twitter, particularly regarding the platform's role in dealing with their depression. Interviews were transcribed and qualitatively analyzed using Atlas.ti software, based on the identification of recurring themes as related to our focus on the development and implications of trust.

\section{Findings}

While Twitter shares certain affordances with other internet platforms characterized by "assumed trust" (de Laat, 2008), our participants were generally more cautious and displayed more "inferred trust." Users described a learning process whereby their initial participation in the social network was based on passive lurking or tweeting faux pas, followed by more "appropriate" participation, characterized mostly by in-the-moment emotional status updates, only after they had been socialized to the platform's dominant modes of engagement. "[At first] I would retweet a lot," explained one user, "and then, like everything in Twitter, a million people would tell you 'don't retweet so much.' So today I try, like, OK, you don't really need to retweet that because you don't want to flood."

While trust was not simply assumed a priori, once inferred it became a central facet of users' perception of Twitter and its unique supportive role in their lives. Users repeatedly contrasted Twitter with other social media, particularly Facebook, portraying Twitter as a counter-public to the more popular-yet-shallow network. As one user explained:

"Facebook feels like a mall, and everything I say there feels like it's in a sea of noise and artificial fluorescent lights. Twitter feels somehow quieter." Users likewise used positively connotated terms such as "home," "lifeline," and "sanctuary" to refer to Twitter. 
Constructed as the "anti-Facebook," Twitter has become a unique echo chamber in which people with depression feel they can present their authentic selves to an empathetic, non-judgmental community of similar outcasts: "On Twitter no one belongs anywhere, but that's the common denominator: not belonging"; "It's a very anarchist network of people with worldviews that are (...) not mainstream"; "It's knowing that there are some people out there, a few, OK, but the few that do understand me and know what I'm talking about."

At the same time, the platform's expectation of profound authenticity paradoxically leads some users to avoid tweeting about positive experiences. "It's hard for me to write about good things that happen to me," said one user. "I'll tell my friends, I don't need to tell Twitter." Another noted sarcastically: "When someone writes about her perfect relationship (...) I'm like, 'hey, lady, it's Twitter here! You're not supposed to write that you're happy. What do you mean, you found love? Delete your account!'" The cordoning off of Twitter as antithetical to the outside world also led some users to experience a crisis of trust when online/offline borders became hazier. As one user explained, "Meeting people from Twitter added a lot to my life but made Twitter itself a space that is less safe."

Finally, a fascinating link emerged between users' psychological sense of social reclusiveness and their sense of political isolation, for which Twitter similarly provided a shared haven. While we did not raise the issue of political identity ourselves in interviews, it was raised by nearly all participants. As one user noted: "[On Twitter] for the first time people around me talked about their emotional states very clearly, and were also very leftist. It was really a support group. Apart from friends I also got a political community." Another user said: "We're a small hotbed of poor leftists there, similar mental conditions [and] openness to different things." In the contemporary Israeli political climate, in which leftists are increasingly ostracized, finding a like-minded, trustworthy community is extremely important. Here too, it is Twitter's lack of popularity that renders it a refuge in which resistant opinions may be expressed with little fear of exposure to the wider public.

\section{References}

de Laat, P. (2008). Online diaries: Reflections on trust, privacy, and exhibitionism. Ethics and Information Technology, 10, 57-69.

Koskela, H. (2004). Webcams, TV shows and mobile phones: Empowering exhibitionism. Surveillance \& Society, 2, 199-215.

Lachmar, E., et al. (2017). \#MyDepressionLooksLike: Examining public discourse about depression on Twitter. JMIR Mental Health, 4(4). https://www.ncbi.nlm.nih.gov/pmc/articles/PMC5666224/

Naslund, J., et al. (2016). The future of mental health care: Peer-to-peer support and social media. Epidemiology and Psychiatric Sciences, 25, 113-122. 
Smith, G. (2016). Theorizing media cultures: The politics of watching and being watched. In H. Tumber \& S. Waisbord (Eds.), The Routledge companion to media and human rights. London: Routledge.

van Uden-Kraan, C., et al. (2008). Empowering processes and outcomes of participation in online support groups for patients with breast cancer, arthritis, or fibromyalgia. Qualitative Health Research, 18, 405-417. 